\title{
Increased RP105-Negative B Cells in IgG4-Related Disease
}

\author{
S. Koarada*, S. Tashiro, N. Nagao, R. Suematsu, A. Ohta and Y. Tada \\ Division of Rheumatology, Faculty of Medicine, Saga University, 5-1-1 Nabeshima, Saga 849-8501, Japan
}

\begin{abstract}
Four patients with IgG4-related disease (IgG4-RD) showed increased percentages of RP105-negative B cells in the peripheral blood. Case 1: A 66-year-old man having retroperitoneal fibrosis had 18.8\% of RP105-negative B cells. Oral prednisolone improved the affected lesions and the percentage of RP105-negative B cells decreased (3.2\%) after the treatment. Case 2: A 53-year-old man with retroperitoneal fibrosis had 27.9\% of RP105-negative B cells. Case 3: A 38year-old man with follicular hyperplasia showed increased percentage of RP105-negative B cells (8.3\%). Case 4: A 60year-old man with interstitial nephritis had $27.5 \%$ of RP105-negative B cells. The treatment decreased the numbers of RP105-negative B cells. Increased numbers of RP105-negatvie B cells is possibly associated with disease activity of IgG4-RD. Analysis of expression of RP105 on B cells may be helpful in evaluation of disease activity of IgG4-RD.
\end{abstract}

Keywords: IgG4-related disease, RP105-negative B cells, plasma cells, plasmablasts.

\section{INTRODUCTION}

IgG4-related disease (IgG4-RD) is a recently recognized rare and novel systemic inflammatory disorder characterized by tumefactive lesions with infiltrating IgG4-positive plasma cells $[1,2]$. IgG4-RD affects the pancreas, retroperitoneal, kidney, biliary tree, aorta, periorbital tissues, lung, meninges, salivary glands, lacrimal glands, and lymph nodes. The elevated serum concentration of IgG4 is closely associated with IgG4-RD. Recent studies have shown that B cell depletion therapy using rituximab (RTX) appears to be an effective and alternative approach in the treatment of refractory IgG4-RD [3]. These results also suggest that B cells may play critical roles in the disease process. However, the etiology of IgG4-RD and B cell biology in the disease have not been fully defined yet. To date, there are a few reports about B cell abnormality in the peripheral blood from patients with IgG4-RD.

RP105 molecule (CD180), one of the toll-like receptor (TLR) associated molecules, is expressed on normal B cells $[4,5]$ and regulates activation of $\mathrm{B}$ cells. In normal persons, RP105-negative B cells are seldom $(1.7 \pm 1.1 \%)$ [6]. Interestingly, in B cell activated diseases, such as systemic lupus erythematosus (SLE), dermatomyositis (DM) and Sjögren's syndrome (SS), the numbers of RP105-negative B cells increase [6,7]. Especially, RP105-negative B cells produce autoantibodies and take part in pathophysiology of human SLE and lupus-prone $(\mathrm{NZB} \times \mathrm{NZW})$ F1 (NZBWF1) mouse [8]. Moreover, detailed analyses have suggested that the RP105-negative B cells are highly activated later B cells including plasmablasts and early plasma cells [9]. Therefore, we investigated the percentages of RP105-negative B cells in the peripheral blood from patients with IgG4-RD. The clinical images of four patients and representative profiles of

*Address correspondence to this author at the Division of Rheumatology, Faculty of Medicine, Saga University, 5-1-1 Nabeshima, Saga 849-8501, Japan; Tel: +81-(952)34-2367; Fax: +81-(952)34-2017;

E-mail: koarada@cc.saga-u.ac.jp flow cytometory are shown in Fig. (1). Blood samples were obtained from four patients. Written informed consent was obtained from all subjects prior to sample acquisition. The study protocol was approved by the Ethics Committees of Saga University. Flow cytometric analysis was performed using FITC-, PE-conjugated anti-human CD180 monoclonal antibodies (mAbs) and APC-conjugated anti-human-CD19 mAbs. The labeled cells were analyzed by a FACScalibur (TM) (Becton Dickinson, Franklin Lakes, NJ, USA).

Case 1: A 66-year-old man presented with fatigue and renal dysfunction (Cr $3.93 \mathrm{mg} / \mathrm{dl})$. The abdomen CT scan revealed hydronephrosis with swelling of soft tissue around the ureter. Gallium scintigraphy showed uptakes of bilateral submandibular glands, kidneys, and periureteral tissue. Biopsy of the left renal pelvis proved dense infiltration of inflammatory cells with fibrosis. Serum IgG4 level was $901 \mathrm{mg} / \mathrm{dl}$. The percentage of RP105-negative B cells was $18.8 \%$. He was diagnosed with retroperitoneal fibrosis. Oral prednisolone (PSL) 30mg/day improved renal function and the tumor-like lesions disappeared. RP105-negative B cells also decreased (3.2\%) after the treatment. Case 2: A 53-yearold man was admitted because of back pain. The abdomen CT scan showed hydronephrosis and MR imaging showed soft tissue swelling around the aorta. In gallium scintigraphy, uptakes around the aorta and kidneys were found. The high level of $\mathrm{IgG} 4(403 \mathrm{mg} / \mathrm{dl})$ and RP105-negative B cells (27.9\%) were found. He was diagnosed with retroperitoneal fibrosis. Oral PSL 30mg/day improved his symptoms promptly. Case 3: A 38-year-old man presented with lymphadenopathy and hypergammaglobulinemia (IgG $5049 \mathrm{mg} / \mathrm{dl})$. Whole body CT scan showed generalized lymphadenopathy. $\mathrm{He}$ was admitted to our hospital. Histological finding of lymph nodes was follicular hyperplasia with germinal center formation and infiltration of plasma cells. Immunohistochemistry of specimen showed that infiltrated cells were IgG4-positvie (IgG4+/IgG+ cells $=50 \%)$. The percentage of RP105-negative B cells was 8.3\%. Case 4: A 60-year-old man was admitted because of 


\section{Case 1}
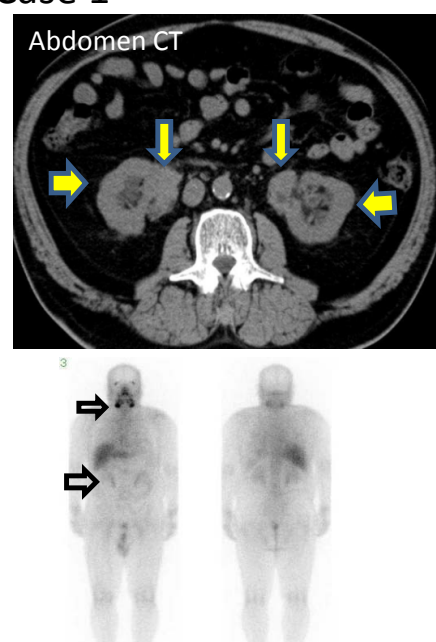

Gallium67 (Ga) scintigraphy

\section{Case 2}

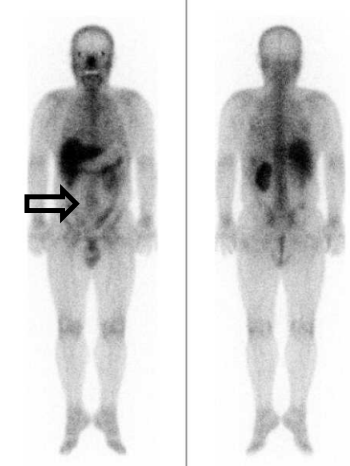

Gallium67 (Ga) scintigraphy

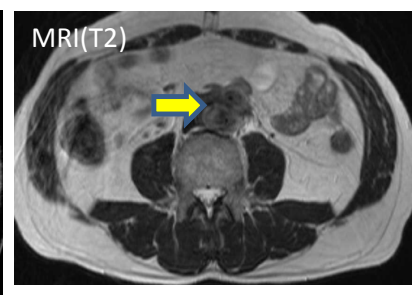

Case 3
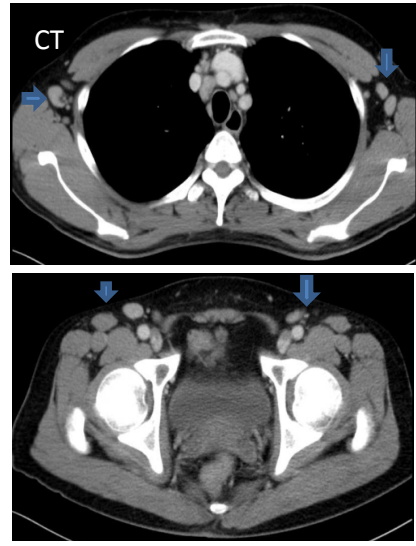

Lymph node

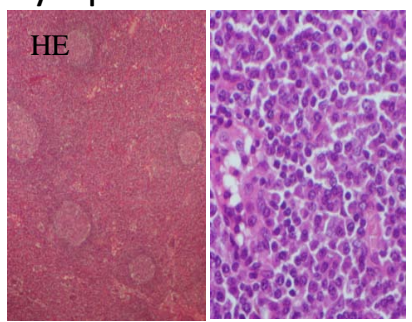

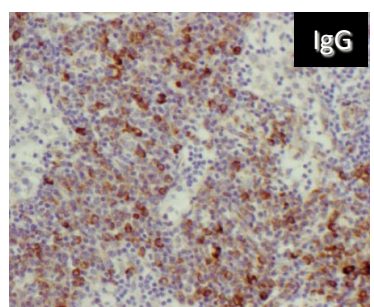

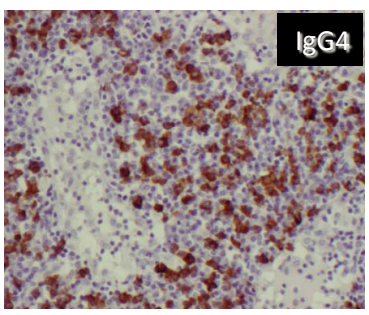

Case 4

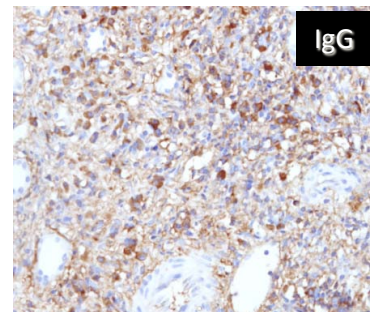

$\lg 6$

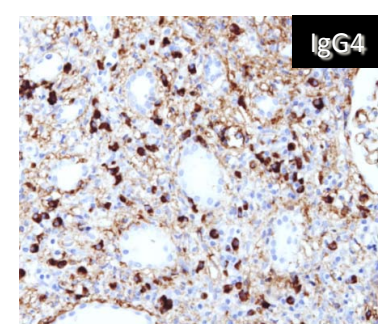

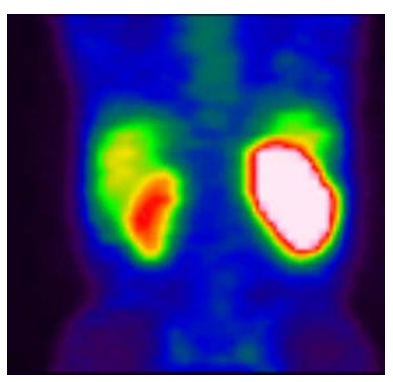

Gallium67 (Ga) scintigraphy

\section{FACS profiles}
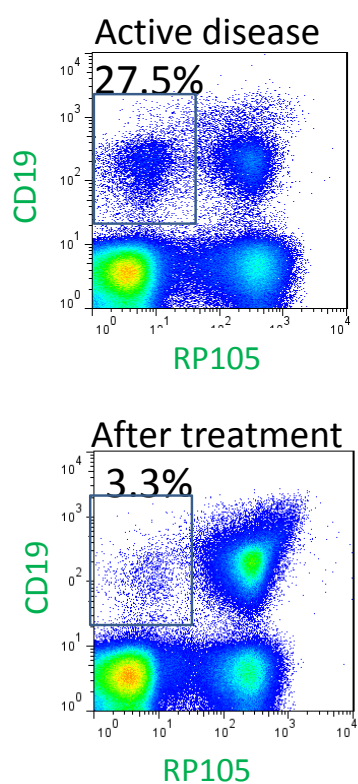

Fig. (1). Case 1: A 66-year-old man with retroperitoneal fibrosis. The abdomen CT scan and the gallium scintigraphy. Case 2: A 53-year-old man with retroperitoneal fibrosis. The gallium scintigraphy and the abdomen MR image (T2-wtighted). Case 3: A 38-year-old man with lymphadenopathy. Whole body CT scan, histological finding and immunohistochemistry (IgG4+/IgG+ cells=50\%) of lymph nodes. Case 4: A 60-year-old man with interstitial nephritis. Immunohistochemistry of the renal biopsy (IgG4+/IgG+ cells > 50\%) and the gallium scintigraphy. The representative flow cytometric profiles and percentages of RP105-negative B cells in peripheral blood before and after the treatment.

renal dysfunction $(\mathrm{Cr} 2.1 \mathrm{mg} / \mathrm{dl})$. Uptakes in gallium scintigraphy were found in kidneys. Renal biopsy proved infiltrated IgG4-postive plasma cells in the specimens (IgG4+/IgG+ cells > 50\%). Serum IgG4 level was $767 \mathrm{mg} / \mathrm{dl}$ and the percentage of RP105-negative B cells was $27.5 \%$. He was diagnosed with interstitial nephritis. Oral PSL $30 \mathrm{mg} /$ day improved renal function $(\mathrm{Cr} 1.36 \mathrm{mg} / \mathrm{dl})$ and the percentage of RP105-negative B cells decreased to $3.3 \%$.

Our results suggest that the RP105-negative B cells increase in IgG4-RD in which activation and dysregulation of B cells may be involved. Because RP105-negative B cells consist of mainly plasmablasts and early plasma cells, precursors of plasma cells may increase in the peripheral blood in IgG4-RD. It is also important to investigate the infiltrating RP105-negative B cells histologically. Moreover, the analyses of the relationship of the numbers of RP105negative B cells with disease activity and severity of organ disorders may be required and we plan further studies.
However, serial analyses of the peripheral blood in two patients after treatment showed that RP105-negative B cells decreased in parallel with disease activity. In addition, interestingly, the percentages of serum level of IgG4 in total IgG may be associated with the percentages of RP105negative B cells (\%IgG4: \%RP105-negative B cells, case 1. 28.3\%: $18.8 \%$, case 2. $20.3 \%: 27.9 \%$, case $3.12 .3 \%: 8.3 \%$, case 4. 25.7\%: 27.5\%). These facts suggest that RP105negative B cells may be one of the possible sources of serum IgG4. Therefore, in IgG4-RD, RP105-negative B cells may be a possible therapeutic target.

We present four cases of IgG4-RD showing increased numbers of RP105-negative B cells. The numbers of RP105negatvie $\mathrm{B}$ cells may be associated with disease activity in evaluated two cases. Analysis of expression of RP105 on B cells may be helpful in evaluation of the patients with IgG4RD. However, its significance in pathophysiology of the disease is still open to further evaluation. 
Syuichi Koarada, M. D., Satoko Tashiro, M.D., Natsumi Nagao, M.D., Rie Suematsu, M. D., Akihide Ohta, M. D., and Yoshifumi Tada, M.D.

Division of Rheumatology, Faculty of Medicine, Saga University, Saga, Japan.

\section{CONFLICT OF INTEREST}

The authors confirm that this article content has no conflict of interest.

\section{ACKNOWLEDGEMENTS}

Financial support for this study was provided by grantaid for scientific research from the Ministry of Education, Science, Sports, and Culture, Japan (No. 22591077). The authors report no conflicts of interest.

\section{REFERENCES}

[1] Stone JH, Zen Y, Deshpande V. IgG4-related disease. N Engl J Med 2012; 366: 539-51.
[2] Umehara H. A new clinical entity: IgG4-related disease (IgG4-RD) discovered in the 21st century. Intern Med 2012; 51: 821-2.

[3] Khosroshahi A, Bloch DB, Deshpande V, Stone JH. Rituximab therapy leads to rapid decline of serum IgG4 levels and prompt clinical improvement in IgG4-related systemic disease. Arthritis Rheum 2010; 62: 1755-62.

[4] Miyake K, Yamashita Y, Hitoshi Y, Takatsu K, Kimoto M. Murine $\mathrm{B}$ cell proliferation and protection from apoptosis with an antibody against a 105-kD molecule: unresponsiveness of X-linked immunodeficient B cells. J Exp Med 1994; 180: 1217-24.

[5] Miura Y, Shimazu R, Miyake K, et al. RP105 is associated with MD-1 and transmits an activation signal in human B cells. Blood 1998; 92: 2815-22.

[6] Koarada S, Tada Y, Ushiyama O, et al. B cells lacking RP105, a novel B cell antigen, in systemic lupus erythematosus. Arthritis Rheum 1999; 42: 2593-600.

[7] Koarada S, Tada Y, Kikuchi Y, et al. CD180 (RP105) in rheumatic diseases. Rheumatology 2001; 40: 1315-6.

[8] Fujita K, Akasaka Y, Kuwabara T, et al. Pathogenesis of lupus-like nephritis through autoimmune antibody produced by CD180 negative B lymphocytes in NZBWF1 mouse. Immunol Lett 2012; 144: $1-6$.

[9] Koarada S, Tada Y, Suematsu R, et al. Phenotyping of P105negative $\mathrm{B}$ cell subsets in patients with systemic lupus erythematosus. Clin Dev Immunol 2012; 2012: 198206.

This is an open access article licensed under the terms of the Creative Commons Attribution Non-Commercial License (http://creativecommons.org/licenses/by-nc/ $3.0 /$ ) which permits unrestricted, non-commercial use, distribution and reproduction in any medium, provided the work is properly cited. 